\title{
Polar Stratospheric Cloud Observations in the 2006/07 Arctic Winter by Using an Improved Micropulse Lidar
}

\author{
Carmen Cordoba-Jabonero, Manuel Gil, and Margarita Yela \\ Atmospheric Research and Instrumentation Branch, Instituto Nacional de Tecnica Aeroespacial, \\ Torrejon de Ardoz, Madrid, Spain \\ MARion MATURILLI AND ROLAND NEUbER \\ Alfred Wegener Institute, Potsdam, Germany
}

(Manuscript received 27 October 2008, in final form 13 May 2009)

\begin{abstract}
The potential of a new improved version of micropulse lidar (MPL-4) on polar stratospheric cloud (PSC) detection is evaluated in the Arctic over $\mathrm{Ny}-\AA$ Alesund $\left(79^{\circ} \mathrm{N}, 12^{\circ} \mathrm{E}\right)$, Norway. The campaign took place from January to February 2007 in the frame of the International Polar Year (IPY) activities. Collocated Alfred Wegener Institute (AWI) Koldewey Aerosol Raman Lidar (KARL) devoted to long-term Arctic PSC monitoring is used for validation purposes. PSC detection is based on lidar retrievals of both backscattering ratio $R$ and volume depolarization ratio $\delta^{V}$. Two episodes were unequivocally attributed to PSCs: 21-22 January and 5-6 February 2007, showing a good correlation between MPL-4 and KARL backscattering ratio datasets (mean correlation coefficient $=0.92 \pm 0.03$ ). PSC layered structures were characterized for four observational periods coincident with KARL measurements. Also, PSC type classification was determined depending on the retrieved $R$ and $\delta^{V}$ values as compared with those obtained by KARL long-term Arctic PSC measurements. Tropospheric cloud cover from lidar observations and both ECMWF potential vorticity and temperature at $475 \mathrm{~K}$, in addition to temperature profiles from AWI daily radiosoundings, are also reported. Height-resolved and temporal evolution of both PSC episodes obtained from MPL-4 measurements clearly show that MPL-4 is a suitable instrument to provide long-term PSC statistic monitoring in polar regions. These results are the first reported on PSC detection in the Arctic by using a low-energy and highly pulsed lidar operating on autonomous and full-time continuous mode MPL-4.
\end{abstract}

\section{Introduction}

Polar stratospheric clouds (PSCs) are recognized as determinant elements for the ozone depletion processes occurring at high latitudes during winter and spring. They provide the surfaces required for a number of heterogeneous catalytic reactions involving air denitrification and $\mathrm{Cl}_{2}$ photolysis, which result on the destruction of stratospheric ozone (see, e.g., Solomon 1999). The volume of air with temperatures below the threshold of PSC condensation is found to be linearly correlated to the magnitude of the ozone losses (Rex et al. 2004, 2006; Tilmes et al. 2004). Therefore, long-

Corresponding author address: Carmen Cordoba-Jabonero, Atmospheric Research and Instrumentation Branch, INTA, Ctra. Ajalvir, km. 4, Torrejon de Ardoz, 28850-Madrid, Spain.

E-mail: cordobajc@inta.es term direct PSC observations provide valuable information on the climatology of the lower stratosphere.

In polar regions, PSCs start to form during winter at stratospheric temperatures below the condensation threshold of the nitric acid trihydrate (NAT), which depends on the water vapor and nitric acid partial pressure (Hanson and Mauersberger 1988). In Antarctica, PSC presence is almost ubiquitous from the beginning of wintertime to early springtime because of very low stratospheric temperatures extending over large areas. On the contrary, Arctic temperatures are close to the threshold of PSC formation, hence both spatial and temporal PSC distributions present a high variability both in day-to-day and in year-to-year scales. In general, they can be observed locally in regions at mesoscale dimensions and just for several days or less. A first threetype PSC widely used classification was proposed by Toon et al. (1990): type I is nitric acid clouds formed 
above the ice frost point, whereas types II and III are water ice optically thin and optically thick, respectively.

Another important characteristic of the PSCs is their low optical depth in comparison with those values usually found for atmospheric aerosols. Typical values of 0.02 have been reported by Tabazadeh et al. (2002) at $0.55 \mu \mathrm{m}$ wavelength, based on the work of McCormick et al. (1981), where a PSC optical depth range of 0.01-0.04 at $1 \mu \mathrm{m}$ is given.

One of the most extended techniques on PSC characterization (detection and type classification) is elastic lidar. In general, instruments of this kind are complex systems requiring intensive attention and good expertise. Both requirements are difficult to fulfill in polar regions.

In the frame of the International Polar Year (IPY), the Spanish Institute for Aerospace Technology [Instituto Nacional de Tecnica Aeroespacial (INTA)] has just deployed in January 2009 a small, easily handled aerosol lidar from the new generation of micropulse lidars [MPL version 4 (MPL-4), Sigma Space Corporation] at Belgrano Station, Antarctica $\left(78^{\circ} \mathrm{S}, 34^{\circ} \mathrm{W}\right)$. Belgrano remains well inside the vortex during wintertime (Parrondo et al. 2007) providing an excellent location for PSC observations. This new equipment will complete the Antarctic program, which INTA is performing continuously from 1994 for stratospheric ozone monitoring and research.

Older versions than MPL-4 are already deployed in polar regions. In Antarctica, a PSC type-II single event was reported at Syowa Station and attributed to low temperature fluctuations related to inertia gravity waves (Shibata et al. 2003). A more extended study has been performed at Amundsen-Scott (South Pole) Station, which unlike the seaside Belgrano Station is located at the Antarctic Plateau (2835 m MSL), where optical conditions for lidar measurements are optimal. In this case, a good data record of $5 \mathrm{yr}$ has been obtained by using the noisier MPL-3 with careful smoothing procedures (Campbell and Sassen 2008). In the Arctic (NyÅlesund), an MPL-3 belonging to the Japanese National Institute of Polar Research (NIPR) is focused on tropospheric aerosols (Shiobara et al. 2003), but to our knowledge no PSC attempt has been carried out to date.

The capability and reliability as well as the limitations on PSC detection of the new MPL-4, which includes a polarization module among other system modifications respect to the previous MPL-3, must be demonstrated. Therefore, the MPL-4 was installed at Koldewey Station, Ny-Ålesund, Spitsbergen, Norway $\left(79^{\circ} \mathrm{N}, 12^{\circ} \mathrm{E}\right)$, from 12 January to 8 February 2007 . The site, frequently inside the polar vortex, was chosen because of the presence of the permanent German Alfred Wegener Institute (AWI) Koldeway Aerosol Raman Lidar (KARL) dedicated to long-term PSC observations from the 1988/89 winter, that was used as reference for comparison purposes.

During the campaign period, two clear PSC episodes were recorded. MPL-4 performance on general characterization (detection and type classification) of these events is the main purpose of this paper, which is organized as follows: in section 2 both MPL-4 and KARL lidar systems are described and the data processing is exposed. Section 3 summarizes the most important highlights on PSC climatology description over $\mathrm{Ny}$ Ålesund obtained from long-term PSC observations performed by KARL (Maturilli et al. 2005; Massoli et al. 2006) to be compared with the final results. Section 4 shows the atmospheric conditions (tropospheric cloud cover and stratospheric meteorology) during the campaign. In section 5, the successful results of the MPL-4 on Arctic PSC detection, including its limitations, are detailed and briefly discussed. Finally, section 6 summarizes the principal conclusions.

\section{Methods}

\section{a. Instrument description}

\section{1) MPL-4}

MPL-4 is an improved version of the standard micropulse lidar (MPL-3) in routine operation within the National Aeronautics and Space Administration (NASA) Micro-Pulse Lidar Network (MPLNET; available online at http://mplnet.gsfc.nasa.gov) and is manufactured by Sigma Space Corporation (Lanham, Maryland; available online at http://www.sigmaspace.com). MPL-4 belongs to INTA.

MPL-4 adds, among the new capabilities, an improved telescope designed for higher stability and a birefrigent polarization module to register the backscattering signal in both parallel (p) and perpendicular (s) channels with respect to the polarized plane of the laser beam. In addition, an MPL presents some features different to a conventional lidar; it is a small, easy-handle system with high autonomy; operational in full-time continuous mode $\left(24 \mathrm{~h} \mathrm{day}^{-1}, 365\right.$ days year $\left.{ }^{-1}\right)$; and presents a high repetition-rate $(2500 \mathrm{~Hz})$ and low-energy $(\sim 10 \mu \mathrm{J})$ " "eye safe" laser as reported by the manufacturer. The system is able to scan the atmosphere up to $30 \mathrm{~km}$ with a good enough signal-to-noise (S-N) relation (Welton et al. 2000; Campbell et al. 2002), well above the heights where PSCs usually appear. The specifications of MPL-4 are summarized in Table 1.

In general, MPL-4 measurements of backscattered signal are performed in 1-min integrated time at $75-\mathrm{m}$ vertical resolution, commuting the polarization control from parallel- (p) to cross-polarized (s) channel each time (i.e., 1-min integrated data are recorded every 
TABLE 1. General MPL-4 and KARL specifications.

\begin{tabular}{llll}
\hline & Lidar system & \multicolumn{1}{c}{ MPL-4 } & KARL \\
\hline Transmitter & Laser & Nd:YLF & Nd:YAG \\
& Wavelength $(\mathrm{nm})$ & 527 & 532 \\
& Pulse repetition rate $(\mathrm{Hz})$ & 2500 & 50 \\
& Pulse energy $(\mathrm{mJ})$ & 0.010 (maximal) & 500 (typical) \\
Receiver (telescope) & Type & Maksutov-Cassegrain & 60 \\
& Diameter $(\mathrm{cm})$ & 18 & Photonian \\
Detector & Type & Avalanche photodiode (APD) & Photocounting \\
& Mode & Photocounting & Polarizing beam splitters \\
Polarization & p and s channels & Birefrigent polarizer module & \\
\hline
\end{tabular}

2 min for each polarization plane). Both $\mathrm{p}$ and $\mathrm{s}$ raw signals must then be corrected by a number of factors affecting the instrument. A complete and detailed description of the correction procedure for the MPL raw signal is found in Campbell et al. (2002). In this sense, monthly calibrations of dark current and afterpulse are performed, whereas overlap is carried out twice a year for a general instrumental checking and maintenance control of the MPL- 4 system. Full overlap is achieved at altitudes higher than $2 \mathrm{~km}$.

\section{2) KARL}

KARL operates in $\mathrm{Ny}-\AA$ Ålesund $\left(79^{\circ} \mathrm{N} 12^{\circ} \mathrm{E}\right)$ by AWI under a long-term PSC monitoring program from the winter of 1988/89 (available online at http://www.awi.de/ en/infrastructure/stations/awipev_arctic_research_base/ data/). The main system is composed of a Nd:YAG laser, with a $50-\mathrm{Hz}$ pulse frequency and $500-\mathrm{mJ}$ (typical) pulse energy, transmitting at $532 \mathrm{~nm}$, and a $\mathrm{XeCl}$ Excimer laser, with $90-\mathrm{Hz}$ pulse frequency, transmitting at 308 $\mathrm{nm}$, and Raman-shifted to $353 \mathrm{~nm}$. Detection channels at 1064, 607, 532 (parallel- and cross-polarized), 385, and $353 \mathrm{~nm}$ are used in photo-counting mode. A mechanical chopper prevents photodetector saturation at low altitudes.

In particular, a vertical resolution of $150 \mathrm{~m}$ was used, and for comparison purposes with MPL observations only the 532-nm p- and s-channel data are used for evaluation. KARL system specifications are shown in Table 1.

\section{b. Data processing}

Stratospheric lidar measurements were limited by tropospheric cloud coverage. Lidar profile ranges below $15 \mathrm{~km}$ and above $30 \mathrm{~km}$ were discarded in this work to avoid possible cirrus cloud signals and instrumental noise, respectively.

Both parallel- (p) and cross-polarized (s) sets of signal profiles were hourly averaged to increase the $\mathrm{S}-\mathrm{N}$ values. In particular, depending on the observational period of time performed by the KARL system, the profiles were averaged in that coincident time interval (further details in section 5).

A Klett-Fernald iterative algorithm (Fernald 1984; Klett 1985) was applied to those averaged registered signal profiles for retrieving the PSC backscattering coefficients. A lidar (extinction to backscattering) ratio of 40 sr (R. Neuber 2006, personal communication), which is commonly used for Arctic PSCs, was introduced in that retrieval algorithm. That selected value is lower than those reported by Reichardt et al. (2004) at $355 \mathrm{~nm}$, according to an expected wavelength dependence of the PSC lidar ratio (backscattering coefficients are retrieved at longer lidar 527-/532-nm wavelengths). In particular, a general version of this algorithm to be used on an MPL is described in Welton et al. (2000). The molecular lidar ratio is known $\left(S_{R}=8 \pi / 3\right)$ and height-resolved molecular backscattering coefficients were calculated from a density profile obtained from daily AWI local meteorological soundings. Retrieved backscattering profiles were used to calculate the backscattering ratio $R$, which is defined as the relation of the total backscattering coefficient (molecular + aerosol) to the molecular backscattering coefficient, as well as the volume depolarization $\delta^{V}(\%)$, which is defined as the ratio of the s-channel to pchannel lidar signals. PSC lidar data were normalized between 25 and $30 \mathrm{~km}$ : backscattering profiles to a backscattering ratio value of $R=1.0$ and depolarization profiles to the molecular value of $0.0144\left(\delta_{\mathrm{MOL}}^{V}=1.44 \%\right)$.

Different PSC types, as observed by both lidar systems, have been classified according to the obtained $R$ and $\delta^{V}$ values (see section 3 for details). For convenience, backscattering ratios $R$ were replaced with the $1-1 / R$ parameter to limit the $R$ values in the range $(0,1)$ for a better graphic representation with the volume depolarization $\delta^{V}$, as suggested by Adachi et al. (2001), adopted by Adriani et al. (2004), and applied by Massoli et al. (2006).

Backscattering ratios above the background stratospheric aerosol $\left(R^{\mathrm{BSA}}=1.15\right)$ were associated with PSC signature for the altitude range of $15-25 \mathrm{~km}$ lasting at least $60 \mathrm{~min}$. According to that definition, two episodes were unequivocally attributed to PSCs: 21-22 January 
TABLE 2. Major characteristics of Arctic PSCs over Ny-Ålesund (Maturilli et al. 2005; Massoli et al. 2006).

\begin{tabular}{|c|c|c|}
\hline Polar occurrence & $\begin{array}{l}5 \text { times higher in } \\
\text { Antarctica than in } \\
\text { the Arctic (Ny- } \AA \text { lesund) }\end{array}$ & $\begin{array}{l}\text { Consequence of a more stable polar vortex, allowing } \\
\text { lower stratospheric temperatures in Antarctica }\end{array}$ \\
\hline PSC type occurrence (\%) & $\begin{array}{l}\text { Liquid STS: } 37 \% \\
\text { Mixed: } 31 \% \\
\text { (Total: } 68 \% \text { ) } \\
\text { Solid NAT: } 25 \% \\
\text { NAT enhanced: } 4 \% \\
\text { NAT "rocks": } 3 \% \\
\text { (Total: } 32 \% \text { ) }\end{array}$ & $\begin{array}{l}\text { Frequent coexistence of different PSC types; occurrence of 'sandwich' } \\
\text { structures in the same vertical profile (Shibata et al. 1999) } \\
\text { Formation of liquid/solid external mixtures: liquid STS with solid } \\
\text { particles (Biele et al. 2001) } \\
0 \% \text { ice; although recently observed but only once on } 26 \text { Jan } 2005 \\
\text { (Maturilli and Dörnbrack 2006) }\end{array}$ \\
\hline Seasonal distribution & $\begin{array}{l}\text { Liquid STS droplets } \\
\text { Solid NAT clouds } \\
\text { Mixed (liquid/solid) clouds }\end{array}$ & $\begin{array}{l}\text { From midwinter (mid-January) onward } \\
\text { Early winter } \\
\text { Over the whole season } \\
\text { Appearance along with NAT and liquid clouds }\end{array}$ \\
\hline Altitude range level & $\begin{array}{l}\text { NAT clouds at lower } \\
\text { altitudes than the } \\
\text { liquid droplets }\end{array}$ & $\begin{array}{l}16-28 \mathrm{~km} \text {, but mainly appearing at } 20-24-\mathrm{km} \text { height } \\
\text { Downward shifting during the season }\end{array}$ \\
\hline Vertical thickness & $\begin{array}{l}\text { LSV PSCs: vertically } \\
\text { thick clouds } \\
\text { SSV PSCs: thin } \\
\text { peak-like structures }\end{array}$ & $\begin{array}{l}\text { Solid NAT clouds and liquid STS droplets } \\
\text { Associated with synoptic temperature conditions } \\
\text { Liquid STS droplets } \\
\text { Connected to local temperature anomalies (mesoscale conditions) }\end{array}$ \\
\hline $\begin{array}{l}\text { Relevance on } \\
\text { ozone depletion }\end{array}$ & Liquid STS droplets & $\begin{array}{l}\text { Direct effect: chlorine activation more efficient than frozen particles } \\
\text { Major significance because they represent a large fraction of the PSC } \\
\text { observations } \\
\text { Indirect effect: induces denitrification }\end{array}$ \\
\hline
\end{tabular}

and 5-6 February, which are also confirmed by KARL measurements, as exposed in section 5 .

\section{Arctic PSC climatology in $\mathrm{Ny}$-Ålesund reported by long-term KARL observations}

A complete and extended Arctic PSC climatology in Ny-Ålesund has been recently reported by Maturilli et al. (2005) and Massoli et al. (2006), where a comparison with the PSC lidar observations in the Antarctic McMurdo Station was also included. Different types of polar stratospheric clouds were historically first identified according to their optical parameters retrieved by lidar: the backscattering ratio $R$ and the volume depolarization ratio $\delta^{V}$ (Browell et al. 1990; Toon et al. 1990). This first PSC classification was split into type I for NAT (subtype Ia) and supercooled ternary solution (STS; subtype-Ib) clouds and type II for ice clouds with quite higher $R$ and $\delta^{V}$ values than those retrieved for the type I. In addition to these types, other PSCs are defined in the literature because of optical measurements not strictly fitting the NAT or STS properties but rather covering a wide range of backscattering and depolarization values (Stein et al. 1999). These PSCs are mainly represented by mixed phases (Shibata et al. 1999; Biele et al. 2001), metastable or transition phases (Tabazadeh and Toon 1996), and NAT-enhanced PSCs (Tsias et al. 1999). For the Arctic, liquid STS PSCs yield major significance in terms of ozone chemistry because they represent a large fraction of the PSC observations. Although solid NAT particles affect ozone chemistry rather indirectly by inducing denitrification, the liquid STS droplets act more directly because of their more efficient chlorine activation rates than frozen particles (Ravishankara and Hanson 1996; Borrmann et al. 1997), which have a larger direct impact for ozone depletion. Arctic ice PSCs are hardly ever observed and are commonly linked to mountain lee waves (e.g., Carslaw et al. 1998). Over Ny-Ålesund, they have been detected only once, on 26 January 2005 (Maturilli and Dörnbrack 2006), and were attributed to unusually strong mesoscale stratospheric temperature anomalies. The most important aspects characterizing PSC presence over NyÅlesund are summarized in Table 2, as extracted from Maturilli et al. (2005) and Massoli et al. (2006).

In particular, based on the complete PSC analysis carried out by Maturilli et al. (2005) and Massoli et al. (2006) in Ny-Ålesund for several winters under a longterm PSC monitoring lidar program, we will adopt the same PSC classification criteria as reported by those works. The corresponding criteria range values for $R$ and $\delta^{V}$ are shown in Table 3 . 
TABLE 3. PSC classification criteria (after Massoli et al. 2006).

\begin{tabular}{cccl}
\hline \hline PSC type (subtype) & Backscattering ratio $R$ & Volume depolarization $\delta^{V}$ & \multicolumn{1}{c}{ Particles } \\
\hline Ia & $R<1.56$ & $10 \%<\delta^{V}<30 \%$ & Solid NAT \\
& $1.25<R<1.56$ & $\delta^{V}<30 \%$ & Solid NAT \\
& $R<1.25$ & $\delta^{V}>30 \%$ & NAT "rocks" \\
& $1.56<R<5$ & $\delta^{V}>20 \%$ & NAT enhanced \\
Ib & $R>1.43$ & $\delta^{V}<3 \%$ & Liquid STS \\
Ib mix (1) & $R<1.43$ & $\delta^{V}<10 \%$ & Mixed (liquid-solid) \\
Ib mix (2) & $1.43<R<1.56$ & $3 \%<\delta^{V}<10 \%$ & Mixed (liquid-solid) \\
Ib mix (3) & $1.56<R<10$ & $3 \%<\delta^{V}<20 \%$ & Mixed (liquid-solid) \\
II & $R>10$ & $\delta^{V}>30 \%$ & Solid ice \\
\hline
\end{tabular}

\section{Atmospheric conditions during the campaign}

\section{a. Tropospheric cloud cover conditions}

PSC detection capabilities depend on tropospheric conditions in terms of cloud cover. Lidar systems reach their optimal performances under clear sky conditions but moderate cloud cover with low optical depths such as cirrus or light fog does not strongly compromise the results. Under these conditions, the signal is only partially screened and PSC detection is still possible. In Fig. 1, a general picture of the cloud cover conditions during the validation campaign (28-day period) is shown on basis of the autonomous full-time MPL-4 performance, unlike the KARL system discrete operational period.

During the overall 28-day campaign, a total cloudless time of $40 \%$ is reported; $5 \%$ of this time corresponds to PSC detection by MPL-4. In addition, a 7\% partial cloud screening frequency is also obtained, but no PSCs were detected during this period. The system was also running under overcast conditions ( $24 \%$ of time), despite limitations of the MPL- 4 capabilities on PSC detection. The rest of time is related to no measurement time (29\%), which corresponds to periods with poor atmospheric conditions for lidar observations (i.e., snowfalls) in which the instrument was switched off. Therefore, the MPL-4 system was operating during the $71 \%$ of the campaign period that was under tropospheric cloud-free, partial cloud screening, and overcast conditions. PSC signature was registered by both lidar systems just under cloudless conditions (no cirrus presence affecting stratospheric lidar returns).

\section{b. Meteorological conditions in the stratosphere}

Temperature fields from the European Centre for Medium-Range Weather Forecasts (ECMWF) indicate that temperatures were low enough to sustain PSCs in the Arctic from mid-November 2006 to late February 2007.

After the formation of the vortex in November 2006, temperatures lower than average prevailed in the Arctic stratosphere throughout most of December. A strong warming occurred in the second half of December. After this event, the vortex cooled down to below the climatological mean, with temperatures low enough to sustain PSCs during January and February 2007, despite minor warming pulses in February (Rösevall et al. 2007).

Figure 2 shows potential vorticity (PV) and temperature maps at the 475- and 550-K potential temperature levels (corresponding approximately to $18.5-19.5$ and $22-23 \mathrm{~km}$, respectively) over the north polar region for the two PSC observed events. Both the vortex edge and the PSC type-I formation threshold at 475 and $550 \mathrm{~K}$ levels are marked (white line): 40 and $100 \mathrm{PVU}$ as values representative of the vortex edge and 195 and $190 \mathrm{~K}$ as theoretical temperatures for PSC type-I formation at the same levels, respectively. In both periods shown in Fig. 2, Ny- ̊̊lesund was deep inside the polar vortex. Planetary wave activity destabilized the polar vortex, creating a second separated, smaller-size vortex center (Rösevall et al. 2007).

This picture depicts the isolation of the polar air mass from air masses outside the polar vortex. Red colors in Fig. 2 (top) are related to very high PV values. Excursions to inner vortex of green filaments (outside vortex air) or even yellow ones (vortex border) are almost lacking.

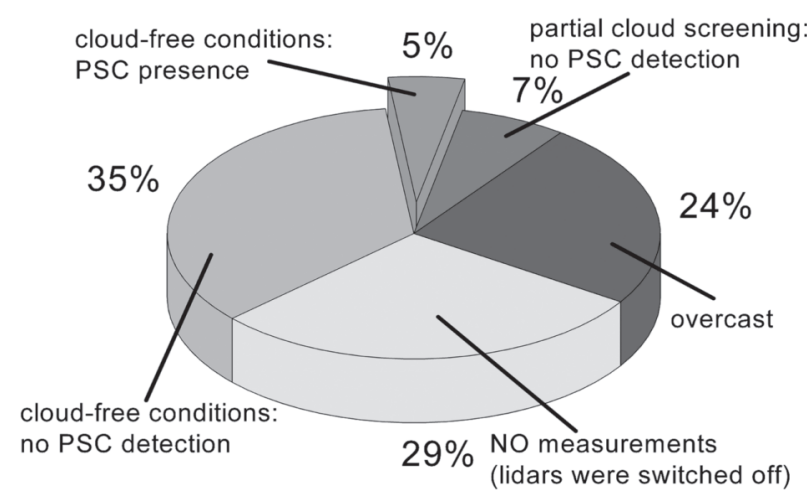

FIG. 1. Cloud cover conditions during the overall PSC observational campaign (28 days) as expressed in measurement time terms (frequency in \%). 


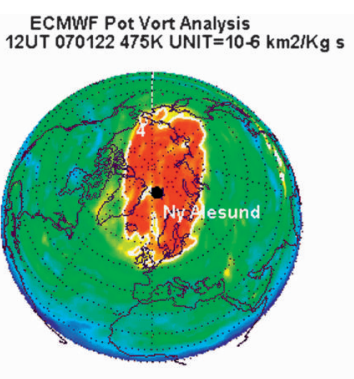

$8 \begin{array}{llllllllll}8 & 2 & 7 & 12 & 1722 & 273338434854\end{array}$

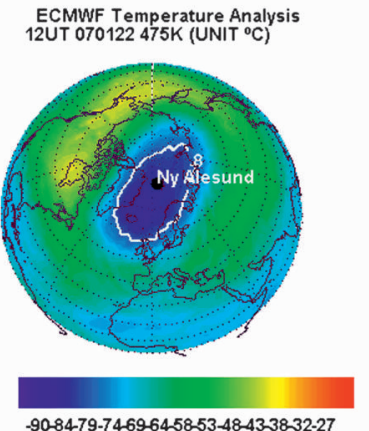

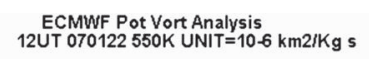

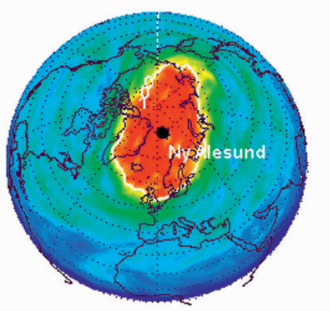

$-111132537496173859710912133$

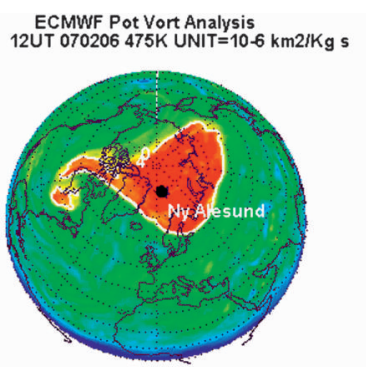

$\begin{array}{llllllllll}8 & 3 & 2 & 721722273338434854\end{array}$
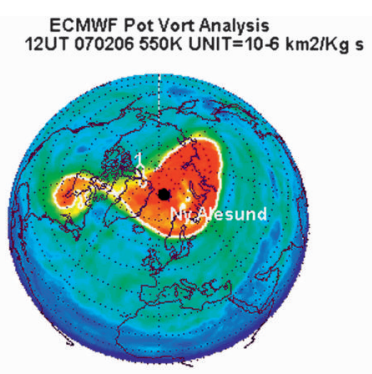

1111325374961738597109121133
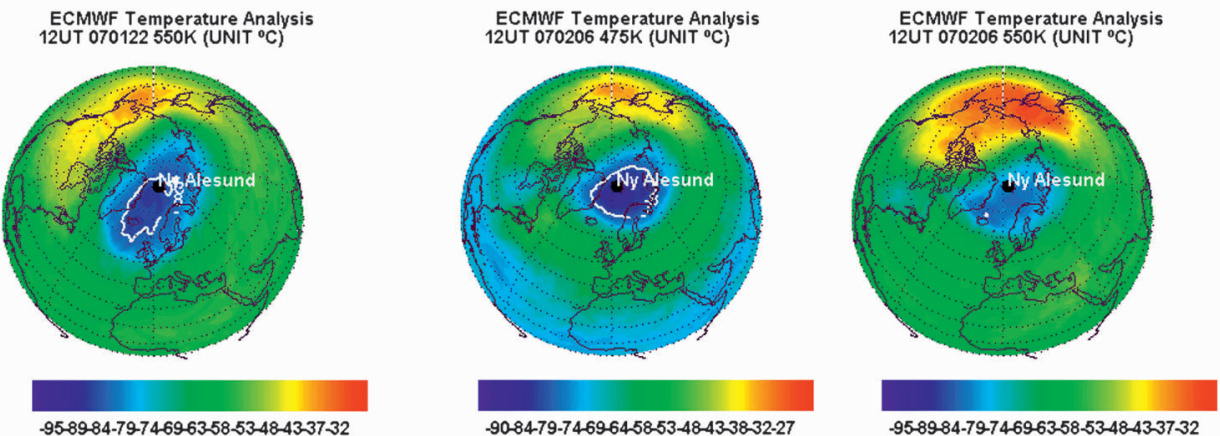

FIG. 2. Maps of (top) PV and (bottom) temperature at the potential temperature levels of 475 and $500 \mathrm{~K}$ over the north polar region (Ny-Ålesund position is marked) for (left) $22 \mathrm{Jan}$ and (right) 6 Feb 2007. The plots are based on data from ECMWF.

In Fig. 3 (top), the temperature at the $475-\mathrm{K}$ isentropic level is displayed. Values were below the theoretical temperature for PSC type I formation during almost all the observational period. PSCs were detected only at temperatures $3 \mathrm{~K}$ below the threshold. PV at the same $475 \mathrm{~K}$ isentropic level (Fig. 3, bottom) has been used as indication of the vortex position with respect to the station. $40 \mathrm{PVU}$ is a value representative of the vortex

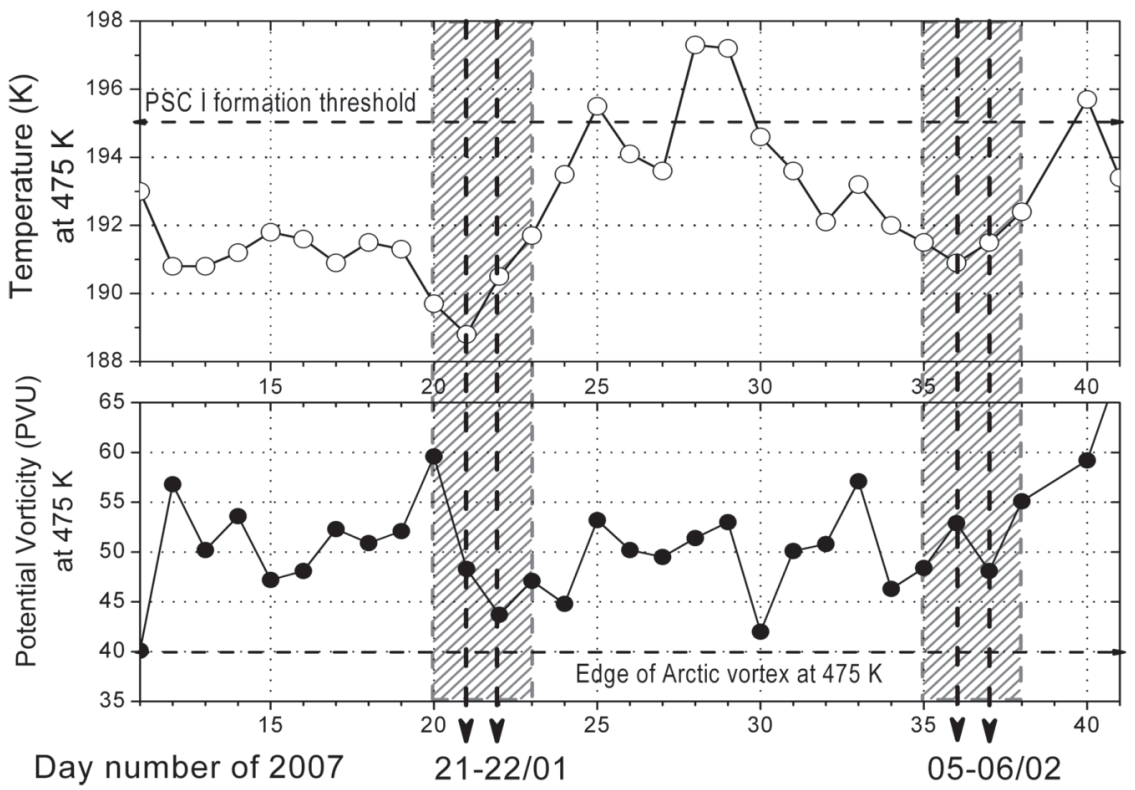

FIG. 3. ECMWF temperature and PV at $475-\mathrm{K}$ level over $\mathrm{Ny}-\AA{ }^{\mathrm{A} l e s u n d}\left(79^{\circ} \mathrm{N}, 12^{\circ} \mathrm{E}\right)$ during the PSC observational campaign. Days with PSC detection are indicated (dashed arrows). 


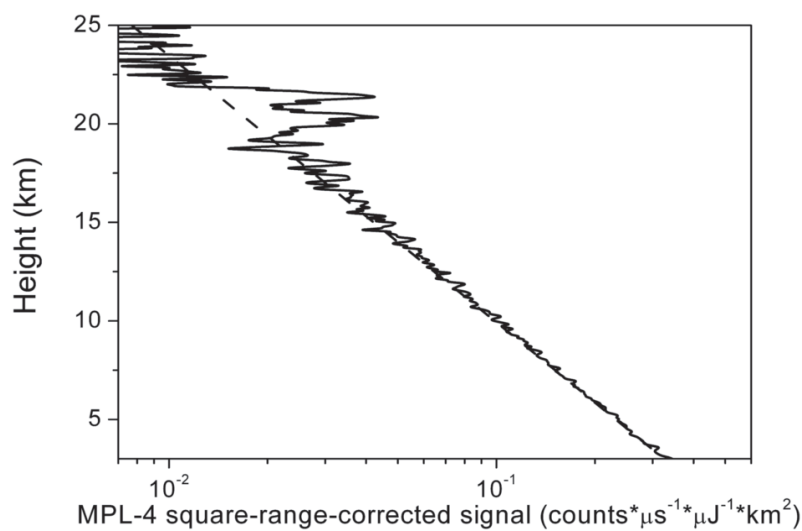

FIG. 4. MPL-4 square-range-corrected backscattered p-channel signal profile for 1-min standard integration time at 0124 UTC 22 Jan 2007, where PSC signature can be clearly distinguished at 19-22-km height.

edge and has been marked as a reference. Results show that $\mathrm{Ny}-\AA$ Allesund was well inside the vortex during the overall campaign.

\section{Results}

MPL-4 square-range-corrected backscattered p-channel signal profile for 1-min standard integration time during a PSC event is shown in Fig. 4 to illustrate how tenuous clouds in the stratosphere can be clearly detected even at short accumulation times. In this particular case, the square-range-corrected lidar signal is increased by a factor of 3 related to the Rayleigh ("aerosol free") reference signal at stratospheric altitudes, showing a clearly distinguished PSC signature at those heights. For general analyses, however, 1-min registered profiles are hourly integrated (see section 2).

\section{a. Correlation between MPL-4 and KARL datasets}

Correlation of the backscattering signals of both lidars has been used as a proxy for MPL-4 capabilities on PSC detection. Four observational periods during the PSC episodes were selected. These periods are chosen depending on the availability of KARL observations, which, on the contrary of MPL-4, was not running in a continuous mode. The periods are as follows: (i) 2-h averaged profile at 2200-2400 UTC 21 January; (ii) 4-h averaged profile at 0000-0400 UTC 22 January; (iii) 2-h averaged profile at 0600-0800 UTC 22 January; and (iv) 3-h averaged profile at 0000-0300 UTC 6 February.

For each of these four periods, the correlation coefficient and its standard deviation between both lidar backscattering ratio datasets are calculated. These calculations were performed for a total of 1000 points in a vertical extension of 10 and $5 \mathrm{~km}$ for the January and
TABLE 4. Correlation coefficients between both lidar backscattering ratio datasets calculated for different observational periods of time.

\begin{tabular}{lccc}
\hline \hline & $\begin{array}{c}\text { Altitude } \\
\text { range } \\
\text { Observational period }\end{array}$ & $\begin{array}{c}\text { Correlation } \\
\text { coefficient } \\
\text { and std dev }\end{array}$ & $\begin{array}{c}\text { Fitting } \\
\text { slope and } \\
\text { std error }\end{array}$ \\
\hline 2200-2400 UTC 21 Jan & $15-25$ & $0.89 \pm 0.10$ & $0.894 \pm 0.002$ \\
0000-0400 UTC 22 Jan & $15-25$ & $0.96 \pm 0.11$ & $0.882 \pm 0.003$ \\
0600-0800 UTC 22 Jan & $15-25$ & $0.91 \pm 0.08$ & $0.941 \pm 0.002$ \\
0000-0300 UTC 6 Feb & $15-20$ & $0.93 \pm 0.11$ & $1.030 \pm 0.003$ \\
\hline
\end{tabular}

February PSC events, respectively. Therefore, the altitude ranges (for details, see Table 4) correspond to vertical resolutions of 10 and $5 \mathrm{~m}$ for each single point in each event. The integration time ranges from 2 to $4 \mathrm{~h}$, depending on the PSC event considered (see Table 4 for details). All these obtained values are shown in Table 4, as well as the fitting slope and its standard error; the altitude range for fitting is also indicated. A mean correlation coefficient of $0.92 \pm 0.03$ is obtained and slopes indicate slightly larger backscattering signals in KARL than in MPL-4 (mean slope $=0.94 \pm 0.06$; for details, see Table 4). In general, a good agreement is found between the MPL-4 and KARL systems. Disagreements are due to small differences in height registering and vertical resolution. This is particularly significant in the backscattering ratio profile on 6 February for a shorter altitude range. Individual profiles will be discussed in detail in section $5 b$.

\section{b. Particular analysis for each PSC event}

\section{1) PSC EVENT ON 21-22 JANUARY 2007}

Profile comparisons of backscattering ratio $R$ for the three periods of simultaneous operation of both lidars are shown in Figs. 5-7 (left panels; thick line for KARL and thin line for MPL-4) for the first episode. A twolayered structure becomes apparent around 20 and $22 \mathrm{~km}$ descending along the day to 19 and $21 \mathrm{~km}$, respectively. This "sandwich" type structure is common in the Arctic PSCs (Shibata et al. 1999; Maturilli et al. 2005; Massoli et al. 2006). The plots in the middle panels of Figs. 5-7 represent the volume depolarization $\delta^{V}$ from KARL observations. Note that the backscattered signal in the MPL-4 s channel does not provide enough S-N ratio for a significant profile retrieval and has not been considered. Improvements in MPL-4 signal analysis processes are ongoing to solve this limitation. Volume depolarization for the pure molecular case $\left(\delta_{\mathrm{MOL}}^{V}=\right.$ $1.44 \%$ ) is also shown as dashed line. The plots in the right panels of Figs. 5-7 display temperature profiles (thick solid line) from local radiosoundings at 1100 UTC. Threshold temperatures for NAT (solid line), STS (dashed 


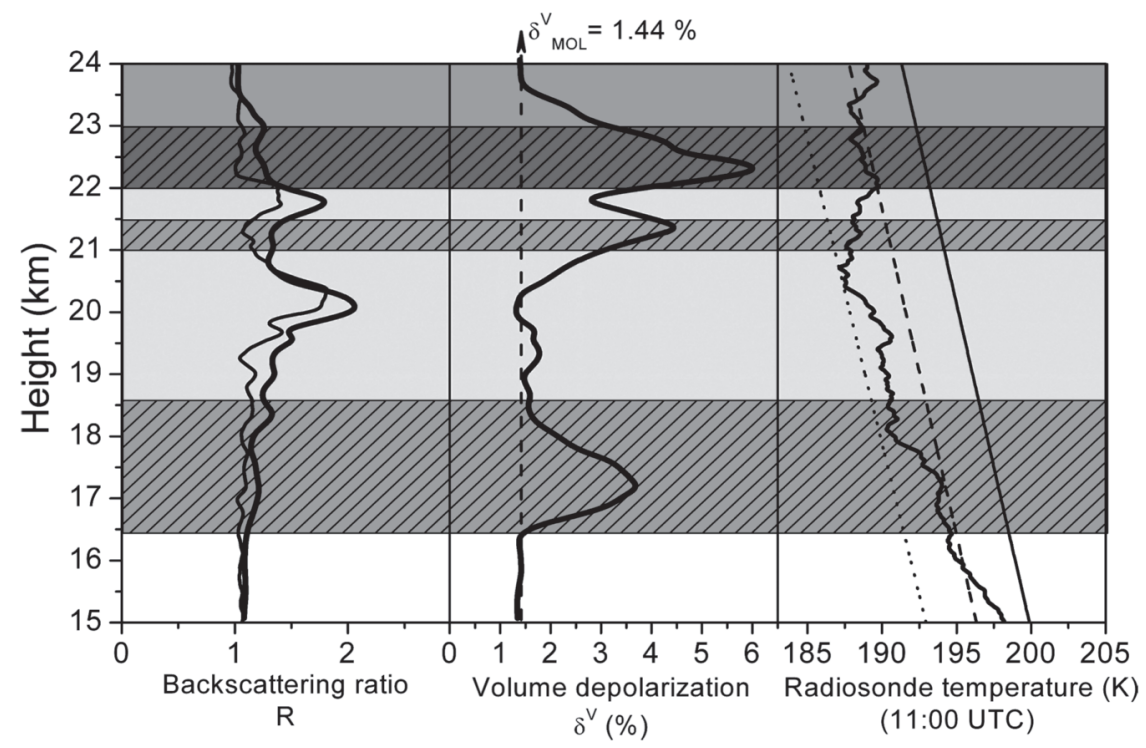

FIG. 5. (left) KARL (thick line) and MPL-4 (thin line) backscattering ratios for the 2-h averaged profile around 2200-2400 UTC 21 Jan. (middle) KARL volume depolarization (thick solid line) and the molecular value $\delta_{\mathrm{MOL}}^{V}=1.44 \%$ (dashed line) for that period. (right) 1100 UTC 21 Jan radiosonde temperature profile (thick solid line) with the 5-ppmv $\mathrm{H}_{2} \mathrm{O}$ and 10-ppbv $\mathrm{HNO}_{3}$ threshold temperatures for NAT (solid line), STS (dashed line), and ice (dotted line). Gray-shaded zones represent types of PSC according to Massoli et al. (2006). For details see text and Table 3 .

line) and ice (dotted line) for 5 ppmv $\mathrm{H}_{2} \mathrm{O}$ and $10 \mathrm{ppbv}$ $\mathrm{HNO}_{3}$ are also shown for reference. Temperature profiles show values below STS all the way above $16-\mathrm{km}$ height with minima at the level of ice threshold.

According to $R$ and $\delta^{V}$ criteria values (see Table 3), each layer has been assigned to a particular PSC type (gray-shaded zones in Figs. 5-7). Liquid STS cloud (PSC type $\mathrm{Ib}$ ) layers are marked in light gray, whereas mixed liquid (PSC type Ib with solid particles) layers are shaded gray or dark gray, depending on the mixed liquid PSC subtype. Line-shaded altitude ranges indicate that $\delta^{V}$ values are lower than those required for solid NAT cloud,

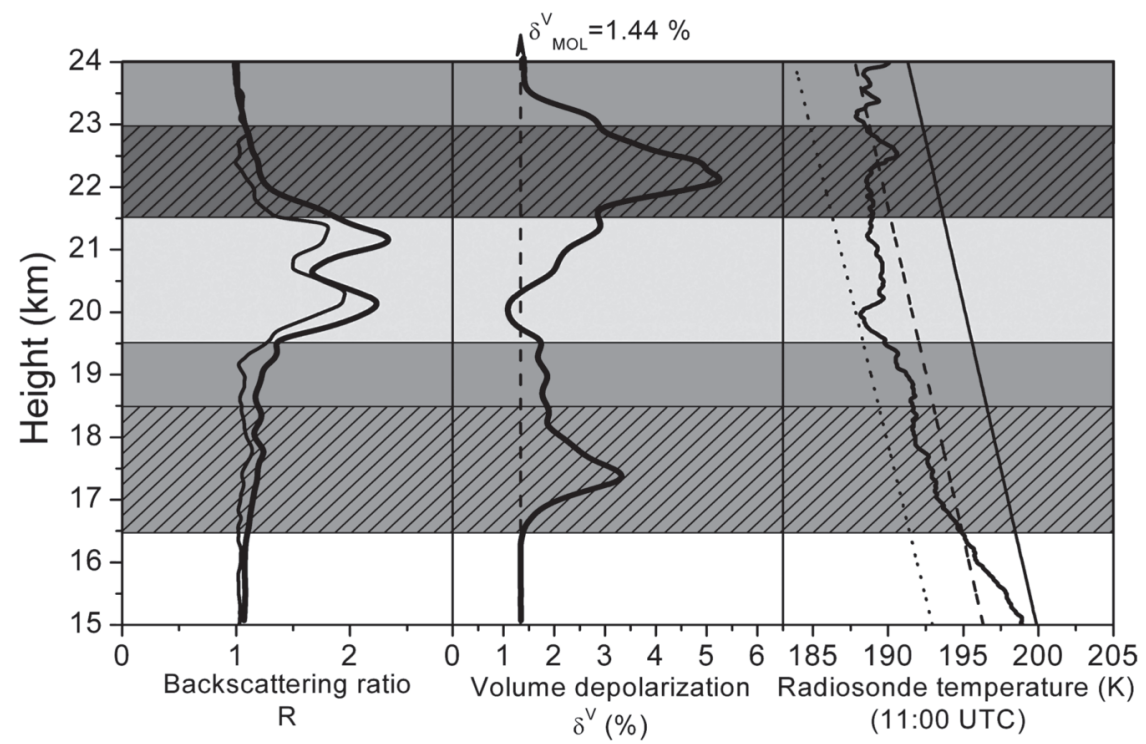

FIG. 6. As in Fig. 5, but (left) for the 4-h averaged profile around 0000-0400 UTC 22 Jan, (middle) for same period as (left), and (right) for 22 Jan. 


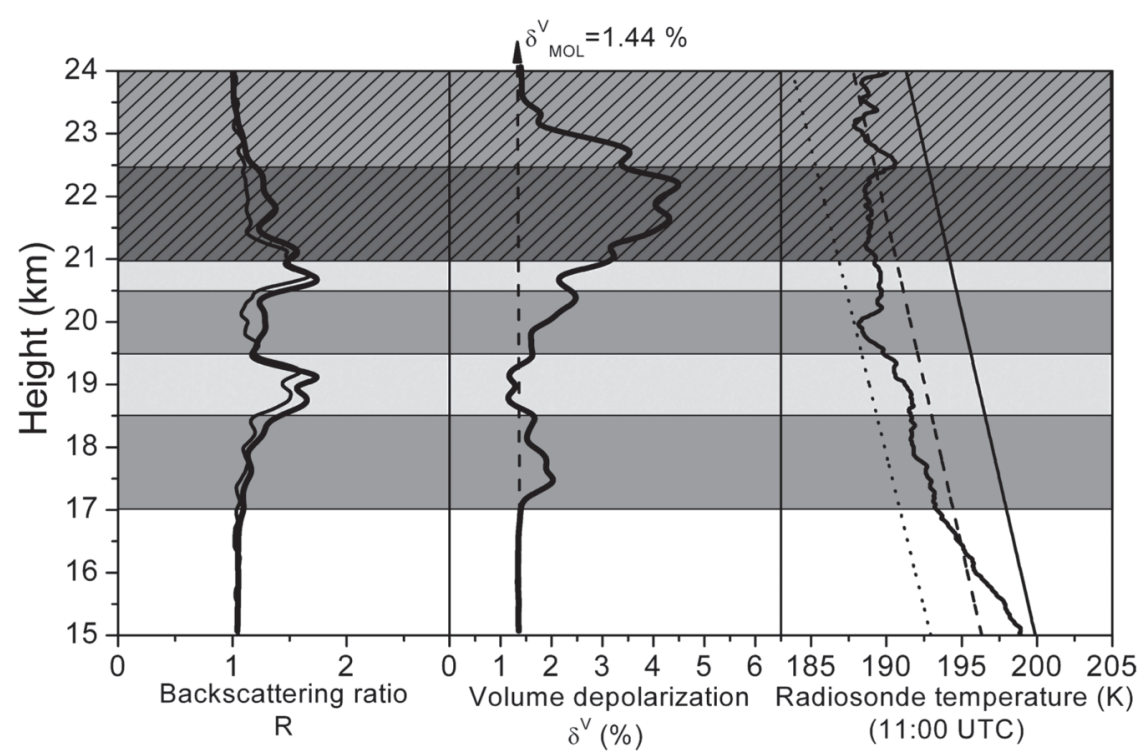

FIG. 7. As in Fig. 5, but (left) for the 2-h averaged profile around 0600-0800 UTC 22 Jan, (middle) for same period as (left), and (right) for $22 \mathrm{Jan}$.

according to the Table 3 classification. Highest backscattering ratios are associated to very low depolarization, which indicates liquid STS in the thickest part of the cloud. Depolarization factors of $3 \%-6 \%$ are observed in the lower and upper boundary of the clouds, where backscattering is less and temperatures are warmer, which is characterized in Table 3 as mixed type-Ib clouds. The relatively high depolarization suggests the presence of a significant fraction of solid NAT particles.

Such a PSC identification, together with the altitude range characteristics (lower height boundary and thickness) for each layer and its corresponding $R$ and $\delta^{V}$ criteria values, are shown in Table 5. As adopted from Massoli et al. (2006), an additional classification is included in dependence on the thickness of each layer; that is, PSC layers are characterized as large-scale variation (LSV) clouds and small-scale variation (SSV) clouds with a threshold value of $1.5-\mathrm{km}$ thickness. In our case, $53 \%$ of the layered structure reported corresponds to LSV (vertically thick) clouds (see Table 2 for a more detailed description).

The complete picture of the evolution of the 2122 January event can be displayed from the MPL-4 continuous dataset (see Fig. 8). Backscattering intensity has been represented in terms of the $1-1 / R$ parameter (for $R$ interpretation, see section 2). Large variability on PSC structure is observed along the overall event. PSCs are already present at the beginning of the measurements at around 2000 UTC 21 January (overcast conditions are reported earlier), extending along the next day (22 January). Maximal backscattering ratio values of $R \sim 3(1-1 / R \sim 0.7)$ are found in the time period of 0800-1100 UTC, corresponding to a persistent narrow layer observed at around $20.5-\mathrm{km}$ height. Unlike the discrete KARL observational periods, MPL-4 can show not only the continuous variations in terms of vertical extension but the detailed time evolution for each PSC episode. PSC behavior looks likely to follow a particular pattern: it is observed during the first hour (which is repeated at a fixed time period), after $8 \mathrm{~h}$, and again (more diffuse) after $16 \mathrm{~h}$, suggesting an influence in the local temperature pattern above the station as downward wave propagating at a rate of about $500 \mathrm{~m} \mathrm{~h}^{-1}$.

\section{2) PSC EVENT ON 5-6 FEBRUARY 2007}

A clear signature of PSC was observed in both lidars again on 5-6 February. Enhanced signals on this time were between $16.0-$ and $19.5-\mathrm{km}$ height. PSCs were present for about $8 \mathrm{~h}$ (from 2000 UTC 5 February to 0400 UTC 6 February).

Radiosonde temperatures are very close or even lower than the STS threshold all the way between 15 and $20 \mathrm{~km}$ (Fig. 9, right). The backscattering ratio profile (Fig. 9, left) also shows a layered structure peaking at lower heights than the previous event. Data are shown for the 3-h averaged profile at 0000-0300 UTC 6 February (for comparison with KARL observational period).

As for the PSC event on 21-22 January, liquid STS cloud (PSC type Ib) layers are marked in light-gray bands, whereas mixed liquid (PSC type Ib with solid particles) layers are gray and dark-gray bands depending on the mixed liquid PSC subtype (see Table 3 for $R$ 
TABLE 5. PSC identification together with the lower height boundary and thickness and the corresponding $R$ and $\delta^{V}$ criteria values for each layer.

\begin{tabular}{|c|c|c|c|c|c|c|}
\hline Time interval & $\begin{array}{l}\text { PSC } \\
\text { layer }\end{array}$ & $\begin{array}{l}\text { Lower height boundary } \\
\text { (thickness) }(\mathrm{km})\end{array}$ & LSV/SSV & $R$ & $\delta^{V}$ & $\begin{array}{l}\text { PSC type } \\
\text { (subtype) }\end{array}$ \\
\hline \multirow[t]{6}{*}{ 2200-2400 UTC 21 Jan } & 1 & $16.5(2.0)$ & LSV & $<1.43$ & $<10 \%$ & Ib mix $(1)^{*}$ \\
\hline & 2 & $18.5(2.5)$ & LSV & $>1.43$ & $<3 \%$ & $\mathrm{Ib}$ \\
\hline & 3 & $21.0(0.5)$ & SSV & $<1.43$ & $<10 \%$ & Ib mix $(1)^{*}$ \\
\hline & 4 & $21.5(0.5)$ & SSV & $>1.43$ & $<3 \%$ & $\mathrm{Ib}$ \\
\hline & 5 & $22.0(1.0)$ & SSV & $1.43-1.56$ & $3 \%-10 \%$ & $\operatorname{Ib} \operatorname{mix}(2)^{*}$ \\
\hline & 6 & $23.0(1.0)$ & SSV & $<1.43$ & $<10 \%$ & Ib mix (1) \\
\hline \multirow[t]{5}{*}{ 0000-0400 UTC 22 Jan } & 1 & $16.5(1.5)$ & LSV & $<1.43$ & $<10 \%$ & Ib mix $(1)^{*}$ \\
\hline & 2 & $18.0(1.5)$ & LSV & $<1.43$ & $<10 \%$ & Ib mix (1) \\
\hline & 3 & $19.5(2.0)$ & LSV & $>1.43$ & $<3 \%$ & $\mathrm{Ib}$ \\
\hline & 4 & $21.5(1.5)$ & LSV & $1.43-1.56$ & $3 \%-10 \%$ & Ib mix $(2)^{*}$ \\
\hline & 5 & $23.0(1.0)$ & SSV & $<1.43$ & $<10 \%$ & Ib mix (1) \\
\hline \multirow[t]{6}{*}{ 0600-0800 UTC 22 Jan } & 1 & $17.0(1.5)$ & LSV & $<1.43$ & $<10 \%$ & Ib mix (1) \\
\hline & 2 & $18.5(1.0)$ & SSV & $>1.43$ & $<3 \%$ & $\mathrm{Ib}$ \\
\hline & 3 & $19.5(1.0)$ & SSV & $<1.43$ & $<10 \%$ & Ib mix (1) \\
\hline & 4 & $20.5(0.5)$ & SSV & $>1.43$ & $<3 \%$ & $\mathrm{Ib}$ \\
\hline & 5 & $21.0(1.5)$ & LSV & $1.43-1.56$ & $3 \%-10 \%$ & Ib mix $(2)^{*}$ \\
\hline & 6 & $22.5(1.5)$ & LSV & $<1.43$ & $<10 \%$ & Ib mix $(1)^{*}$ \\
\hline \multirow[t]{5}{*}{ 0000-0300 UTC 6 Feb } & 1 & $16.0(0.5)$ & SSV & $<1.43$ & $<10 \%$ & Ib mix (1) \\
\hline & 2 & $16.5(1.5)$ & LSV & $>1.43$ & $<3 \%$ & $\mathrm{Ib}$ \\
\hline & 3 & $18.0(0.3)$ & SSV & $<1.43$ & $<10 \%$ & Ib mix (1) \\
\hline & 4 & $18.3(0.5)$ & SSV & $>1.43$ & $<3 \%$ & $\mathrm{Ib}$ \\
\hline & 5 & $18.8(0.7)$ & SSV & $<1.43$ & $<10 \%$ & Ib mix (1) \\
\hline
\end{tabular}

* Layer where the retrieved $\delta^{V}$ value is slightly lower than that required for NAT cloud layer classification.

and $\delta^{V}$ criteria values). In this case, KARL-retrieved volume depolarization ratio $\delta^{V}$ is lower than $\delta_{\mathrm{MOL}}^{V}$ values (dashed line shown in Fig. 9, middle) in the overall PSC detection range. Therefore, this layered structure could be related to spherical liquid particles (PSC type Ib) with only narrow layers presenting liquid/ solid particle mixture characteristics. Particular PSC type (and subtype) identification, as well as its altitude range and the corresponding $R$ and $\delta^{V}$ criteria values, is shown for each layer in Table 5. Unlike the previous PSC event, $80 \%$ of layered structure in this case corresponds to SSV clouds (for further details, see Table 2).

The temporal and height-resolved evolution of this PSC episode for 5-6 February is shown in Fig. 10 by using the full-time MPL dataset (color mapping of the $1-1 / R$ parameter is the same as for 21-22 January). Backscattering ratio values $R \sim 2.0-3.0(1-1 / R \sim 0.5-0.7)$ at 2100-2400 UTC 5 February are found at around 19.0$19.5-\mathrm{km}$ height. At around 0000 UTC, this PSC vanishes and a feeble one appears at a lower altitude (around $17.0-18.0 \mathrm{~km})$ with values of $R \sim 1.8-2.0(1-1 / R \sim 0.5)$ lasting for $4 \mathrm{~h}$.

\section{Conclusions}

First results are presented on PSC backscatter detection in the Arctic at Ny-Ålesund, Norway $\left(79^{\circ} \mathrm{N}, 12^{\circ} \mathrm{E}\right)$, by using a low-energy, highly pulsed, and easily handled micropulse lidar (MPL-4) operating on autonomous and full-time continuous mode. Results are compared for validation with those retrieved by the AWI Koldewey Aerosol Raman Lidar (KARL) system, which is routinely in operation for long-term Arctic PSC monitoring (available online at http://www.awi.de/en/infrastructure/ stations/awipev_arctic_research_base/data/).

MPL-4 has demonstrated its capability for PSC detection even at moderate backscattering signals in spite of its low-energy laser pulse. PSC signature is detected even at the standard 1-min integration time. A good agreement is found in intensity and height-resolved limits with the KARL system. However, in the current state for analysis retrieval of the observed PSC events, the S-N ratio in the cross-polarized channel is too low at those PSC altitudes and at the integration times used during the campaign, which substantially decreases the capabilities for PSC type identification. Improvements in signal analysis processes are ongoing to solve this limitation.

MPL-4 can be operated in an almost unattended and full-time ( $24 \mathrm{~h} \mathrm{day}^{-1}, 365$ days year ${ }^{-1}$ ) mode, retrieving the overall evolution of the clouds in both altitude and time progression. This particular MPL- 4 feature allows a fine characterization at time scales, which is lost when using discrete periods. Therefore, despite the fact that MPL-4 performance on PSC detection is partially fulfilled, 

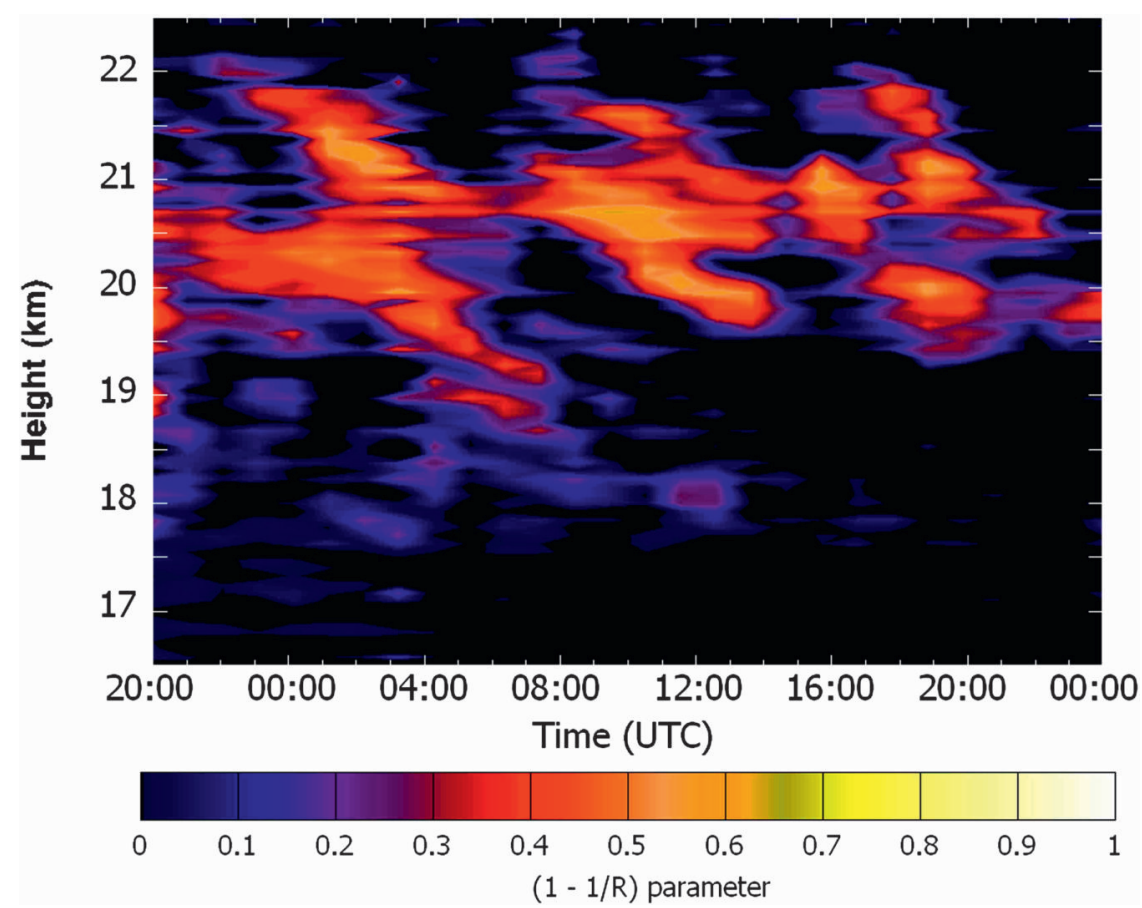

FIG. 8. PSC height-resolved and temporal evolution on 21-22 Jan 2007 in Ny-Ålesund on the basis of the $1-1 / R$ parameter obtained from MPL-4 measurements. Profiles are hourly integrated with a vertical resolution of $75 \mathrm{~m}$.

only for the backscattering ratio, at this stage, this kind of lidar system can obviously provide a valuable characterization of polar clouds, being a suitable instrument to provide long-term PSC statistics in polar regions.
The INTA MPL-4 instrument has been installed at Belgrano Station $\left(78^{\circ} \mathrm{S}, 34^{\circ} \mathrm{W}\right)$ in early January 2009 for the next Antarctic campaign within the frame of the Ozone Layer and UV Radiation in a Changing Climate

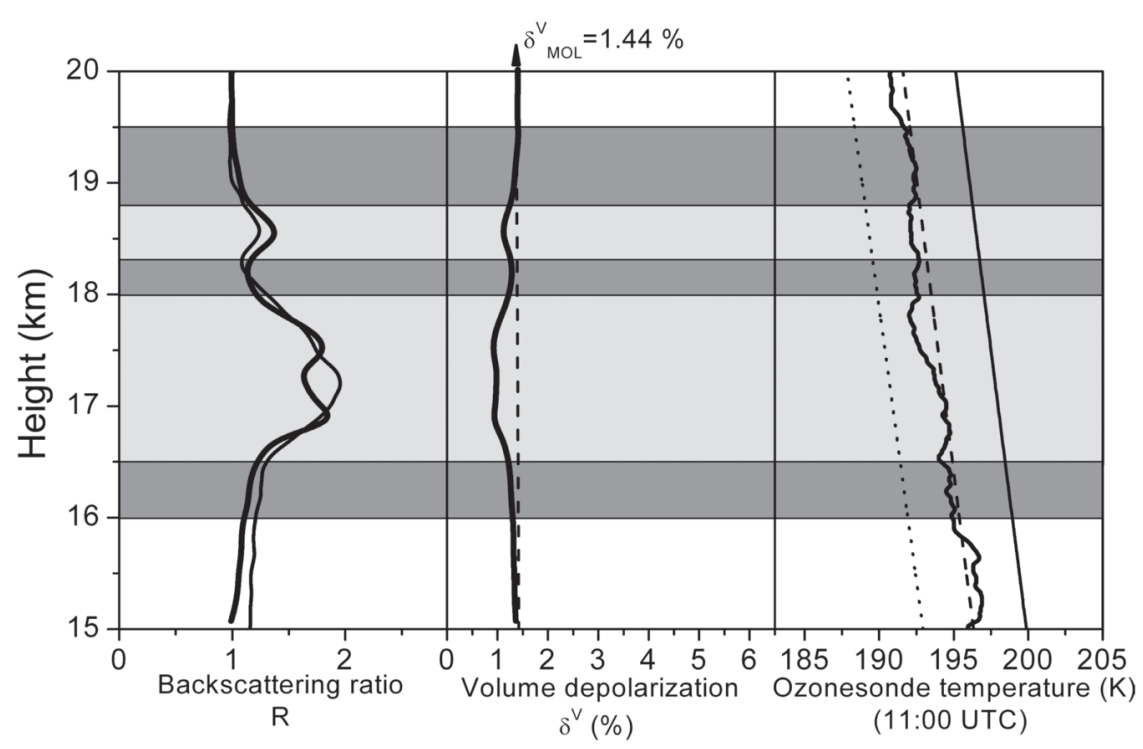

FIG. 9. As in Fig. 5, but (left) for the 3-h averaged profile around 0000-0300 UTC 6 Feb, (middle) for same period as (left), and (right) for 1100 UTC 6 Feb. Temperature profile in (right) is from ozonesonde (radiosonde data not available). 


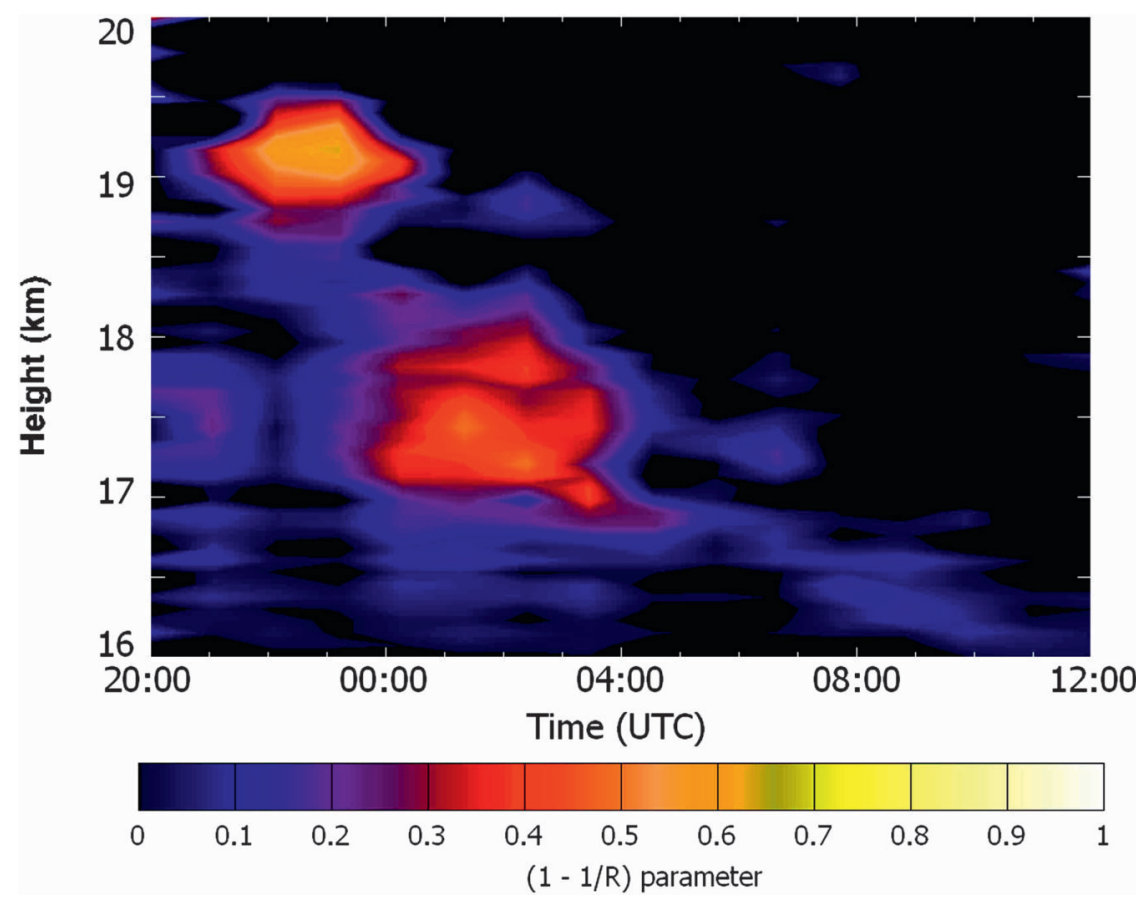

FIG. 10. As in Fig. 8, but for 5-6 Feb 2007.

Evaluated during IPY (ORACLE-O3) project (available online at http://www.awi-potsdam.de/www-pot/ atmo/ORACLE-O3/).

Acknowledgments. We thank the French-German AWIPEV Station team at Ny-Ålesund (Norway) for their wholehearted assistance and support and for performing lidar and radiosonde measurements. The authors also want to thank the anonymous reviewer for numerous helpful comments and suggestions. We thank the European Centre for Medium-Range Weather Forecasts (ECMWF) for providing the meteorological data. We are also grateful to I. Alonso (INTA) for his help during the campaign and M. Navarro and M. Parrondo (INTA) for their help in IDL figure format treatment and ECMWF data management. This work has been supported by INTA Project IGE03004. C. C.-J. holds a contract through Spanish I+D+i Plan, Project CGL2004-03669/CLI.

\section{REFERENCES}

Adachi, H., T. Shibata, Y. Iwasaka, and M. Fujiwara, 2001: Calibration method for the lidar-observed stratospheric depolarization ratio in the presence of liquid aerosol particles. Appl. Opt., 40, 6587-6595.

Adriani, A., P. Massoli, G. Di Donfrancesco, F. Cairo, M. L. Moriconi, and M. Snels, 2004: Climatology of polar stratospheric clouds based on lidar observations from 1993 to 2001 over McMurdo station, Antarctica. J. Geophys. Res., 109, D24211, doi:10.1029/2004JD004800.
Biele, J., A. Tsias, B. P. Luo, K. S. Carslaw, R. Neuber, G. Beyerle, and T. Peter, 2001: Non-equilibrium coexistence of solid and liquid particles in Arctic stratospheric clouds. J. Geophys. Res., 106, 22 991-23 007.

Borrmann, S., S. Solomon, J. E. Dye, D. Baumgardner, K. K. Kelly, and K. R. Chan, 1997: Heterogeneous reactions on stratospheric background aerosols, volcanic sulfuric acid droplets, and type I polar stratospheric clouds: Effects of temperature fluctuations and differences in particle phase.J. Geophys. Res., 102, 3639-3648.

Browell, E. V., and Coauthors, 1990: Airborne lidar observations in the wintertime Arctic stratosphere: Polar stratospheric clouds. Geophys. Res. Lett., 17, 385-388.

Campbell, J. R., and K. Sassen, 2008: Polar stratospheric clouds at the South Pole from five years of continuous lidar data: Macrophysical, optical, and thermodynamic properties. J. Geophys. Res., 113, D20204, doi:10.1029/2007JD009680. , D. L. Hlavka, E. J. Welton, C. J. Flynn, D. D. Turner, J. D. Spinhirne, V. S. Scott III, and I. H. Hwang, 2002: Fulltime, eye-safe cloud and aerosol lidar observation at Atmospheric Radiation Measurement program sites: Instruments and data processing. J. Atmos. Oceanic Technol., 19, 431-442.

Carslaw, K. S., and Coauthors, 1998: Increased stratospheric ozone depletion due to mountain-induced atmospheric waves. Nature, 391, 675-678.

Fernald, F. G., 1984: Analysis of atmospheric lidar observations: Some comments. Appl. Opt., 23, 652-653.

Hanson, D. R., and K. Mauersberger, 1988: Laboratory studies of the nitric acid trihydrate: Implications for the south polar stratosphere. Geophys. Res. Lett., 15, 855-858.

Klett, J. D., 1985: Lidar inversion with variable backscatter/ extinction ratios. Appl. Opt., 24, 1638-1643.

Massoli, P., M. Maturilli, and R. Neuber, 2006: Climatology of Arctic polar stratospheric clouds as measured by lidar in 
Ny-Ålesund, Spitsbergen $\left(79^{\circ} \mathrm{N}, 12^{\circ} \mathrm{E}\right)$. J. Geophys. Res., 111, D09206, doi:10.1029/2005JD005840.

Maturilli, M., and A. Dörnbrack, 2006: Polar stratospheric ice cloud above Spitsbergen. J. Geophys. Res., 111, D18210, doi:10.1029/2005JD006967.

— R. Neuber, P. Massoli, F. Cairo, A. Adriani, M. L. Moriconi, and G. Di Donfrancesco, 2005: Differences in Arctic and Antarctic PSC occurrence as observed by lidar in Ny- $\AA$ lesund $\left(79^{\circ} \mathrm{N}, 12^{\circ} \mathrm{E}\right)$ and McMurdo $\left(78^{\circ} \mathrm{S}, 167^{\circ} \mathrm{E}\right)$. Atmos. Chem. Phys., 5, 2081-2090.

McCormick, M. P., and Coauthors, 1981: High-latitude stratospheric aerosols measured by the SAM II satellite system in 1978 and 1979. Science, 214, 328-331.

Parrondo, M. C., M. Yela, M. Gil, P. von der Gathen, and H. Ochoa, 2007: Mid-winter lower stratosphere temperatures in the Antarctic vortex: Comparison between observations and ECMWF and NCEP operational models. Atmos. Chem. Phys., 7, 435-441.

Ravishankara, A. R., and D. R. Hanson, 1996: Differences in the reactivity of Type I polar stratospheric clouds depending on their phase. J. Geophys. Res., 101, 3885-3890.

Reichardt, J., A. Dörnbrack, S. Reichardt, P. Yang, and T. J. McGee, 2004: Mountain wave PSC dynamics and microphysics from ground-based lidar measurements and meteorological modeling. Atmos. Chem. Phys., 4, 1149-1165.

Rex, M., R. J. Salawitch, P. von der Gathen, N. R. P. Harris, M. P. Chipperfield, and B. Naujokat, 2004: Arctic ozone loss and climate change. Geophys. Res. Lett., 31, L04116, doi:10.1029/2003GL018844.

— stratospheric ozone loss and climate change. Geophys. Res. Lett., 33, L23808, doi:10.1029/2006GL026731.

Rösevall, J. D., D. P. Murtagh, and J. Urban, 2007: Ozone depletion in the 2006/2007 Arctic winter. Geophys. Res. Lett., 34, L21809, doi:10.1029/2007GL030620.

Shibata, T., K. Shiraishi, H. Adachi, Y. Iwasaka, and M. Fujiwara, 1999: On the lidar-observed sandwich structure of polar stratospheric clouds (PSCs). 1. Implications for the mixing state of the PSC particles. J. Geophys. Res., 104, 21 603-21 611.

— K. Sato, H. Kobayashi, M. Yabuki, and M. Shiobara, 2003: Antarctic polar stratospheric clouds under temperature perturbation by nonorographic inertia gravity waves observed by micropulse lidar at Syowa Station. J. Geophys. Res., 108, 4105, doi:10.1029/2002JD002713.

Shiobara, M., M. Yabuki, and H. Kobayashi, 2003: A polar cloud analysis based on Micro-pulse Lidar measurements at $\mathrm{Ny}$ Ålesund, Svalbard and Syowa, Antarctica. Phys. Chem. Earth, 28, 1205-1212.

Solomon, S., 1999: Stratospheric ozone depletion: A review of concepts and history. Rev. Geophys., 37, 275-316.

Stein, B., and Coauthors, 1999: Optical classification, existence temperatures, and coexistence of different polar stratospheric cloud types. J. Geophys. Res., 104, 23 983-23 993.

Tabazadeh, A., and O. B. Toon, 1996: The presence of metastable $\mathrm{HNO}_{3} / \mathrm{H}_{2} \mathrm{O}$ solid phases in the stratosphere inferred from ER2 data. J. Geophys. Res., 101, 9071-9078.

_ K. Drdla, M. R. Schoeberl, P. Hamill, and O. B. Toon, 2002: Arctic "ozone hole" in a cold volcanic stratosphere. Proc. Natl. Acad. Sci. USA, 99, 2609-2612.

Tilmes, S., R. Müller, J.-U. Grooß, and J. M. Russell, 2004: Ozone loss and chlorine activation in the Arctic winters 1991-2003 derived with the tracer-tracer correlations. Atmos. Chem. Phys., 4, 2181-2213.

Toon, O. B., E. V. Browell, S. Kinne, and J. Jordan, 1990: An analysis of lidar observations of polar stratospheric clouds. Geophys. Res. Lett., 17, 393-396.

Tsias, A., and Coauthors, 1999: Aircraft lidar observations of an enhanced type Ia polar stratospheric clouds during APEPOLECAT. J. Geophys. Res., 104, 23 961-23 969.

Welton, E. J., and Coauthors, 2000: Ground-based lidar measurements of aerosols during ACE-2: Instrument description, results, and comparisons with other ground-based and airborne measurements. Tellus, 52B, 636-651. 